\title{
Influence of atomic order on the enthalpy of formation and bulk modulus of the sigma
}

\author{
phase \\ LIU Wei ${ }^{1,2}$, LU Xiao-Gang ${ }^{1,3, *}$, BOULET Pascal ${ }^{2}$, RECORD Marie-Christine ${ }^{4, *}$, HU Qing-Miao ${ }^{5}$ \\ ${ }^{1}$ School of Materials Science and Engineering, Shanghai University, 200444 Shanghai, P.R. China \\ ${ }^{2}$ Aix-Marseille Univ, CNRS, MADIREL, Marseille, France \\ ${ }^{4}$ Aix-Marseille Univ, Université de Toulon, CNRS, IM2NP, Marseille, France \\ 5 Titanium Alloy Laboratory, Institute of Metal Research, Chinese Academy of Sciences, 72 Wenhua Road, Shenyang \\ 110016, China \\ *Corresponding author: xglu@t.shu.edu.cn (LU Xiao-Gang) and m-c.record@univ-amu.fr (RECORD Marie-Christine) \\ tianziben@163.com (wei.liu@etu.univ-amu.fr) (tianziben@shu.edu.cn) \\ pascal.boulet@univ-amu.fr \\ qmhu@imr.ac.cn
}

Abstract

We have calculated the enthalpy of formation and bulk modulus for 19 typical binary sigma compounds with different atomic order (i.e. atomic constituent distribution or site occupancy preference on inequivalent sites of a crystal structure) based on the experimental site occupancy as well as completely ordered and hypothetically disordered states by using the EMTO-CPA (Exact Muffin-Tin Orbitals - Coherent Potential Approximation) method. The calculation results show that at $0 \mathrm{~K}$ the sigma phase in ordered state bears a lower enthalpy of formation and a larger bulk modulus than the ones in less ordered state.

Key words: sigma phase, atomic order, enthalpy of formation, bulk modulus, EMTO-CPA

1. Introduction

The topologically close-packed (TCP) phases are one of the largest groups of intermetallic compounds, which are composed of metallic atoms with different atomic size. These atoms adapt each other forming a very complex crystal structure with high space utilization and high coordination number $(\mathrm{CN})$. The characteristic of the structure is that it stacked by coordination polyhedron of $\mathrm{CN} 12,14,15$ and 16 [1]. TCP phase is common precipitation in Fe-, Ni-, Co- based superalloys and austenitic and duplex stainless steels [2-5]. The common members of TCP phases are sigma, chi, mu, laves, A15, R, P, delta and M phases [1,2]. A small amount of TCP precipitations can cause the effect of precipitation hardening and grain boundary strengthening [3,6]. However, in most cases, TCP phase is a detrimental phase in alloys. It is hard and brittle, which make alloys crack under the service conditions in tensile stress. Moreover, the TCP precipitation will lead to poor alloying elements in matrix, and thus decreases the mechanical properties and corrosion resistance of materials [2,3]. Therefore, accurate prediction and reasonable control of the precipitation of TCP phase is obviously the key to alloy design of iron-based, nickel-based, cobalt-based superalloys and stainless steels.

The sigma phase is a typical example of TCP phases, which is with large homogeneity range and designated as tetragonal 
structure (space group $D_{4 t h}^{14}-\mathrm{P} 42 / \mathrm{mnm}$ ) with 30 atoms distributed on five inequivalent sites [7,8] as presented in Table 1 . It can serve as a prototype of TCP phases. In the present work, the EMTO_CPA method was conducted to reveal the influence of atomic order (i.e. atomic constituent distribution or site occupancy preference on inequivalent sites of a crystal structure) on the enthalpy of formation and the bulk modulus of the sigma phase. To facilitate explanation, the ordered and disordered state is designated with atomic occupancy as presented in Table 1. All the binary sigma phase systems studied were designated as A-B where atom A holds a larger atomic size than atom B.

Table 1. Crystal structure of the sigma phase with atomic occupancy corresponding to the ordered and disordered compounds $\mathrm{A}_{2} \mathrm{~B}$ and $A_{x} B_{(1-x)}\left(V_{A}>V_{B}\right.$, where $V_{A}$ and $V_{B}$ are the molar volumes of the constitutive elements in their hypothetic sigma phase structure from

\begin{tabular}{llllll}
\multicolumn{5}{c}{ the CALPHAD assessments [9]). } \\
\hline Space group & $\mathrm{P} 4_{2} / \mathrm{mnm}(\mathrm{no} .136)$ & & & \\
Wyckoff position & $2 \mathrm{a}$ & $4 \mathrm{f}$ & $8 \mathrm{i}_{1}$ & $8 \mathrm{i}_{2}$ & $8 \mathrm{j}$ \\
Coordination number $(\mathrm{CN})$ & 12 & 15 & 14 & 12 & 14 \\
Ordered state $\left(\mathrm{A}_{2} \mathrm{~B}\right)$ & $\mathrm{B}$ & $\mathrm{A}$ & $\mathrm{A}$ & $\mathrm{B}$ & $\mathrm{A}$ \\
Disordered state $\left(\mathrm{A}_{\mathrm{x}} \mathrm{B}_{(1-\mathrm{x})}\right)$ & $\mathrm{x} \cdot \mathrm{A}$ & $\mathrm{x} \cdot \mathrm{A}$ & $\mathrm{x} \cdot \mathrm{A}$ & $\mathrm{x} \cdot \mathrm{A}$ & $\mathrm{x} \cdot \mathrm{A}$ \\
\hline
\end{tabular}

2. Methodology and calculation details

The first-principles calculations were performed using the exact muffin-tin orbitals (EMTO) method [10,11], with which, the Green's function technique is used to solve the one electron Kohn-Sham equation. The optimized overlapping muffintin approximation is used when dealing with the effective potential in the one-electron equation. Besides, the total energy is corrected with the full charge density (FCD) method [10]. The wave function is expanded by using the basis sets of the exact muffin-tin orbitals $[10,11]$. The coherent potential approximation (CPA) [12-14] is incorporated within the EMTO code.

Green's function has been calculated for 16 complex energy points distributed exponentially on a semicircular contour. We adopted the scalar-relativistic and soft-core approximations. The electronic exchange-correlation functional within the generalized-gradient approximation (GGA) as parametrized by Perdew et al. [15] has been used. The Brillouin zone is sampled by a uniform $\mathrm{k}$-point mesh $(3 \times 3 \times 6)$ without any smearing technique.

3. Results and discussion

3.1. Influence of atomic order on enthalpy of formation 

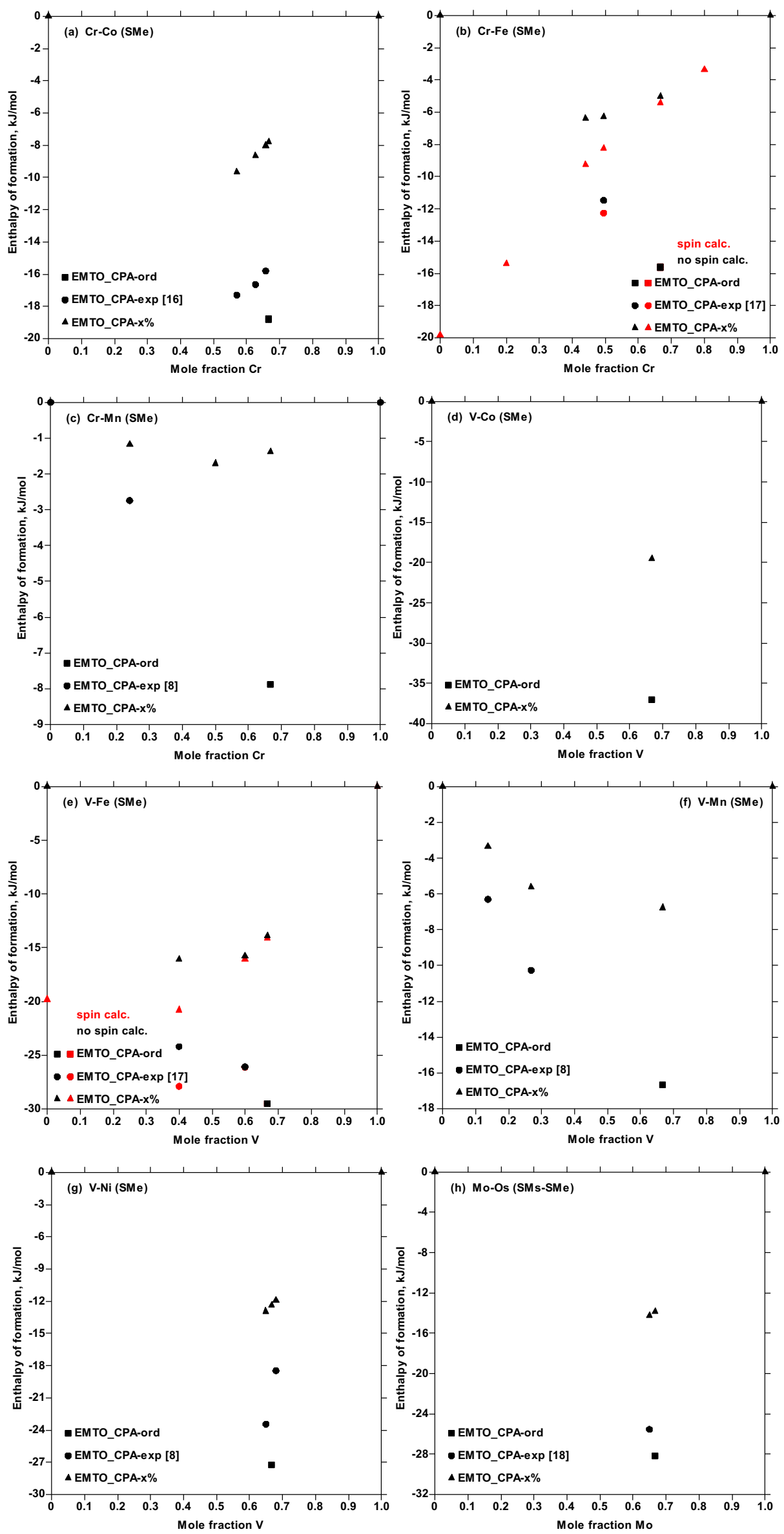

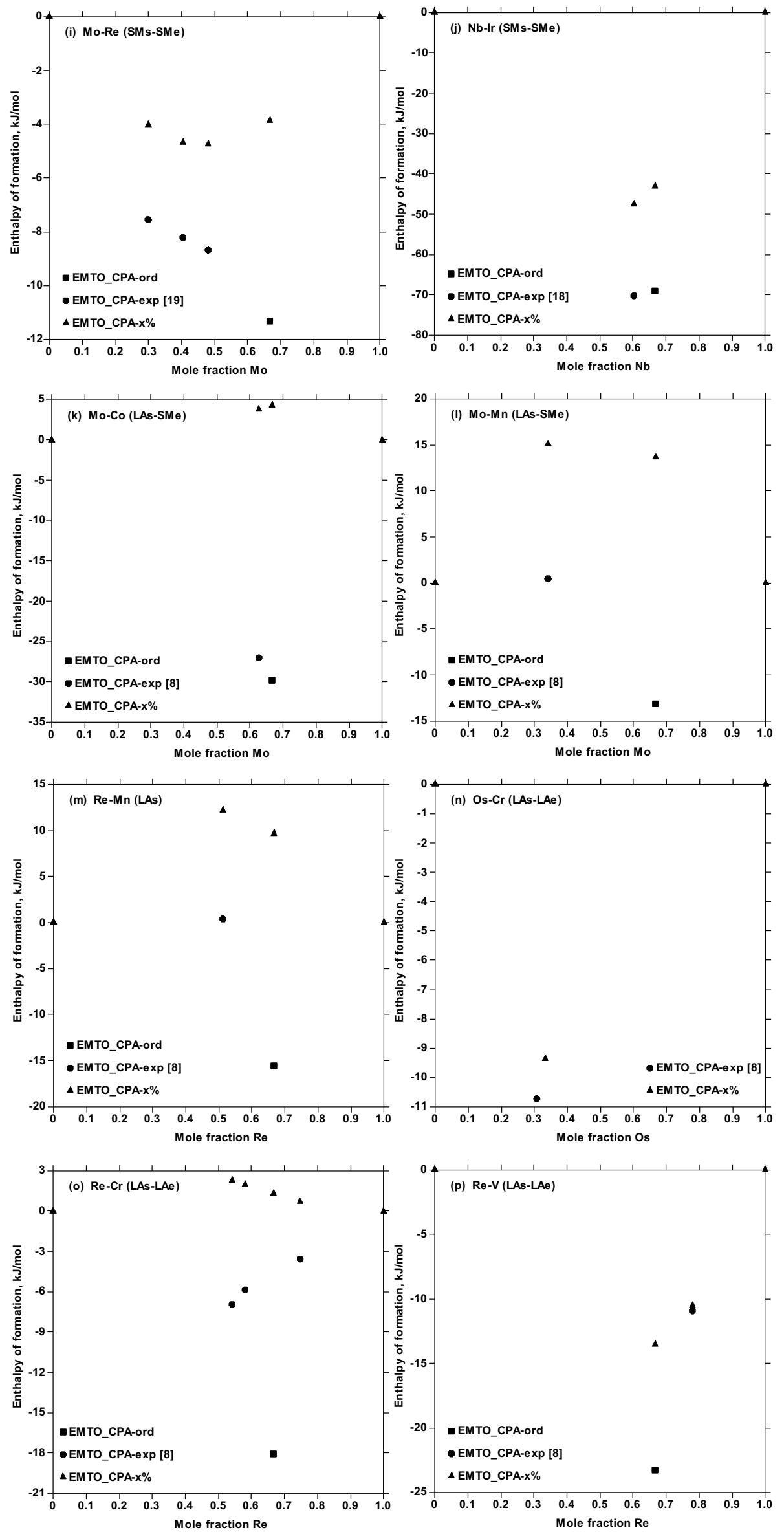

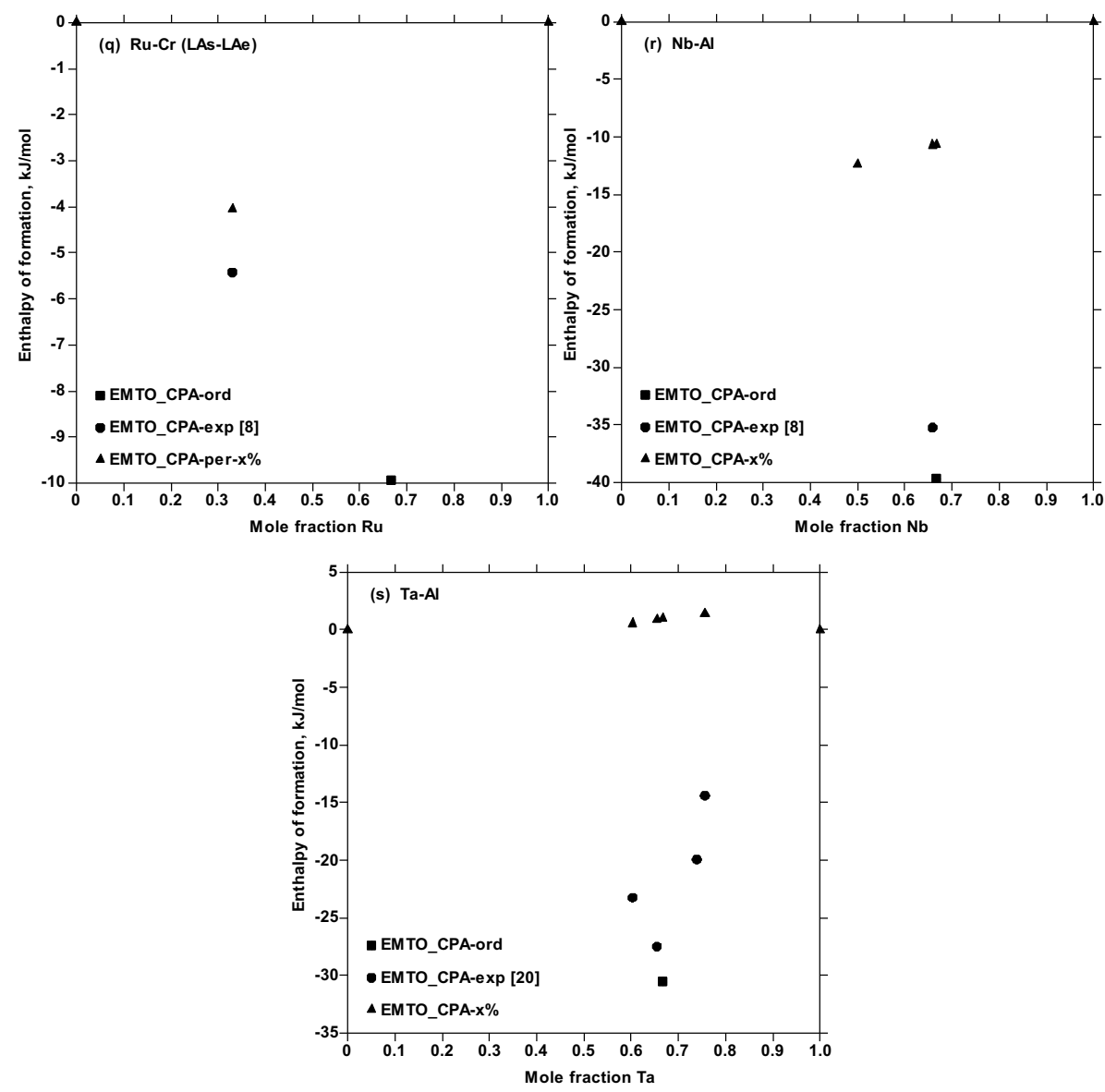

Fig. 1. Enthalpy of formation of A-B binary sigma phase systems referred to the enthalpy of the pure elements in a hypothetic sigma phase structure calculated by EMTO-CPA method at $0 \mathrm{~K}$.

Fig. 1 presents the enthalpy of formation of the sigma phase calculated by using the EMTO_CPA method. EMTO-CPA-ord, EMTO-CPA-exp and EMTO-CPA- $\mathrm{x} \%$ represent results for enthalpy calculated with site occupancies corresponding to that of completely ordered state, experimental measurements [8,16-20] and completely disordered state, respectively. The binary systems of interest are Cr-Co, Cr-Fe, Cr-Mn, Mo-Co, Mo-Mn, Mo-Os, Mo-Re, Nb-Al, Nb-Ir, Os-Cr, Re-Cr, Re-Mn, Re-V, Ru-Cr, Ta-Al, V$\mathrm{Co}, \mathrm{V}-\mathrm{Fe}, \mathrm{V}-\mathrm{Mn}$ and V-Ni binary systems.

Based on Liu et al.'s work [21], these systems have been labelled SMe, SMs-SMe, LAs-SMe, LAs, LAs-LAe or X-Al $(\mathrm{X}=\mathrm{Nb}, \mathrm{Ta})$ according to the difference in total number of electron shells and total number of valence electrons between $\mathrm{A}$ and B atoms (see Fig.1). LAs and LAe mean that atom A has larger total number of electron shells and larger number of valence electrons than atom B, respectively. SMs and SMe mean that atom A has smaller total number of electron shells and smaller number of valence electrons than atom B, respectively. The calculation results indicate that the sigma compound in a more ordered state holds a lower enthalpy of formation (more negative), i.e. the ordered structure is more stable than the less ordered ones at $0 \mathrm{~K}$.

Besides, it has been found out that the formation enthalpy of the sigma phase depends on both the volume difference and the electron configuration of its constitutive elements. Table 2 presents the volume difference between the two constitutive elements (A and B) and the enthalpy of formation of the $\mathrm{A}_{66.7} \mathrm{~B}_{33.3}$ sigma phase in ordered and disordered states calculated by EMTO-CPA method at $0 \mathrm{~K}$. 
It indicates that for SMe and SMs-SMe categories, generally, the enthalpy of formation for compounds in both ordered and disordered states decreases with increasing the size difference between the two constitutive elements (except for V-Fe and V-Ni systems between which inversion is observed). For LAs and LAs-LAe categories, the enthalpy of formation in disordered state for systems with large size difference (Re-Mn and Re-Cr systems), is positive, which indicates a less stable state. This behavior is related to the fact that the effects of the size and electrons factors (LAs or LAe factors) on atomic order for these categories are contradictory see Ref. [21]. On the one hand, by considering the size factor, atom A tends to occupy large $\mathrm{CN}$ sites, namely $4 \mathrm{f}, 8 \mathrm{i}, 8 \mathrm{j}$; on the other hand, by considering LAs (i.e. Atom A is with the larger total number of electron shells than atom B) or LAe (i.e. Atom A is with the larger number of valence electrons than atom B) factors, atom $\mathrm{A}$ tends to occupy small $\mathrm{CN}$ sites, namely $2 \mathrm{a}, 8 \mathrm{i}_{2}$. For LAs-SMe category, due to the influence of LAs factor and the large size difference, for both Mo-Co and Mo-Mn systems, the enthalpy of formation in disordered state is also positive.

Moreover, the enthalpy of formation for Mo-Co system is smaller than that for Mo-Mn system, due to the large effect of $\mathrm{SMe}$ factor (i.e. Atom $\mathrm{A}$ is with the smaller number of valence electrons than atom B, which makes atom $\mathrm{A}$ tend to occupy large $\mathrm{CN}$ sites [21]) for Mo-Co system reduces the effect of LAs factor on atomic order. By considering electron loss and gain, $\mathrm{X}-\mathrm{Al}(\mathrm{X}=\mathrm{Nb}, \mathrm{Ta})$ category behave similarly as LAs-SMe category [21]. The strong effect of LAs factor and a relatively large size difference for Ta-Al system cause the enthalpy of formation in disordered state to be positive.

In addition, calculations considering magnetism were conducted for $\mathrm{Cr}-\mathrm{Fe}$ and V-Fe systems as presented in Fig. 1 (b, e). The calculation results show that at $0 \mathrm{~K}$ when magnetism is accounted for compounds are more stable than those for which magnetism is not accounted for, especially for compounds with high content in $\mathrm{Fe}$, which is agree with the experimental measurements $[22,23]$.

Table 2. Size difference between the constitutive elements (A and B) and enthalpy of formation (Hf) of the $A_{66.7} B_{33.3}$ sigma phase in both ordered and disordered states calculated by EMTO-CPA method at $0 \mathrm{~K}$. $\mathrm{V}_{\mathrm{A}}$ and $\mathrm{V}_{\mathrm{B}}$ are the molar volumes of element $\mathrm{A}$ and $\mathrm{B}$ in their hypothetic sigma phase structure from the CALPHAD assessments [9].

\begin{tabular}{|c|c|c|c|c|c|c|c|c|c|}
\hline \multirow{2}{*}{ category } & \multirow{2}{*}{ A-B } & \multirow{2}{*}{$\begin{array}{c}\left(\mathrm{V}_{\mathrm{A}^{-}}\right. \\
\left.\mathrm{V}_{\mathrm{B}}\right) / \mathrm{V}_{\mathrm{A}}\end{array}$} & \multicolumn{2}{|c|}{$\mathrm{Hf}, \mathrm{kJ} / \mathrm{mol}$} & \multirow{2}{*}{ category } & \multirow{2}{*}{ A-B } & \multirow{2}{*}{$\begin{array}{l}\left(\mathrm{V}_{\mathrm{A}^{-}}\right. \\
\left.\mathrm{V}_{\mathrm{B}}\right) / \mathrm{V}_{\mathrm{A}}\end{array}$} & \multicolumn{2}{|c|}{$\mathrm{Hf}, \mathrm{kJ} / \mathrm{mol}$} \\
\hline & & & ordered & disordered & & & & ordered & disordered \\
\hline $\mathrm{SMe}$ & $\begin{array}{l}\mathrm{Cr}- \\
\mathrm{Mn}\end{array}$ & 0.0147 & -7.88 & -1.37 & $\begin{array}{l}\text { SMs- } \\
\text { SMe }\end{array}$ & $\mathrm{Nb}-\mathrm{Ir}$ & 0.2126 & -69.05 & -43.13 \\
\hline $\mathrm{SMe}$ & $\mathrm{Cr}-\mathrm{Fe}$ & 0.0588 & -15.62 & -5.04 & $\begin{array}{l}\text { LAs- } \\
\text { SMe }\end{array}$ & $\begin{array}{l}\text { Mo- } \\
\text { Mn }\end{array}$ & 0.2386 & -13.08 & 13.68 \\
\hline $\mathrm{SMe}$ & $\begin{array}{l}\mathrm{V}- \\
\mathrm{Mn}\end{array}$ & 0.1052 & -16.67 & -6.77 & $\begin{array}{l}\text { LAs- } \\
\text { SMe }\end{array}$ & $\begin{array}{l}\text { Mo- } \\
\text { Co }\end{array}$ & 0.3087 & -29.83 & 4.27 \\
\hline $\mathrm{SMe}$ & $\begin{array}{l}\mathrm{Cr}- \\
\mathrm{Co}\end{array}$ & 0.1053 & -18.79 & -7.79 & LAs & $\begin{array}{l}\text { Re- } \\
\text { Mn }\end{array}$ & 0.2006 & -15.53 & 9.68 \\
\hline $\mathrm{SMe}$ & $\mathrm{V}-\mathrm{Fe}$ & 0.1453 & -29.54 & -13.90 & $\begin{array}{l}\text { LAs- } \\
\text { LAe }\end{array}$ & $\mathrm{Re}-\mathrm{V}$ & 0.1065 & -23.29 & -13.54 \\
\hline $\mathrm{SMe}$ & $\mathrm{V}-\mathrm{Ni}$ & 0.1739 & -27.26 & -12.35 & $\begin{array}{l}\text { LAs- } \\
\text { LAe }\end{array}$ & $\begin{array}{c}\mathrm{Ru}- \\
\mathrm{Cr}\end{array}$ & 0.1298 & -9.94 & \\
\hline $\mathrm{SMe}$ & V-Co & 0.1876 & -37.06 & -19.56 & $\begin{array}{l}\text { LAs- } \\
\text { LAe }\end{array}$ & $\begin{array}{l}\mathrm{Re}- \\
\mathrm{Cr}\end{array}$ & 0.1887 & -18.09 & 1.32 \\
\hline $\begin{array}{l}\text { SMs- } \\
\text { SMe }\end{array}$ & $\begin{array}{l}\text { Mo- } \\
\operatorname{Re}\end{array}$ & 0.0476 & -11.33 & -3.87 & $\mathrm{X}-\mathrm{Al}$ & $\begin{array}{c}\mathrm{Nb}- \\
\mathrm{Al}\end{array}$ & 0.143 & -39.69 & -10.63 \\
\hline
\end{tabular}




\begin{tabular}{rrrrrrrrrr} 
SMs- & Mo- & & & & & Ta- & & & \\
SMe & Os & 0.0856 & -28.18 & -13.85 & X-Al & Al & 0.1454 & -30.54 & 0.97 \\
\hline
\end{tabular}

3.2 Influence of atomic order on bulk modulus

The bulk modulus of the sigma phase in the $\mathrm{Cr}-\mathrm{Co}, \mathrm{Cr}-\mathrm{Fe}, \mathrm{Cr}-\mathrm{Mn}, \mathrm{Mo}-\mathrm{Co}, \mathrm{Mo}-\mathrm{Fe}, \mathrm{Mo}-\mathrm{Mn}, \mathrm{Mo}-\mathrm{Os}$, Mo- $\mathrm{Re}, \mathrm{Nb}-\mathrm{Al}, \mathrm{Nb}-$ Ir, Re-Cr, Re-Mn, Ta-Al, V-Co, V-Fe, V-Mn and V-Ni systems has also been evaluated by fitting the Birch-Murnaghan equation of state [24,25]. The corresponding results are listed in Table 3. For most systems, sigma phase in ordered state has a large bulk modulus and a small molar volume, which indicates a strong binding between atoms and a compact structure, respectively. Only for Mo-Re system, sigma phase in ordered state has a large bulk modulus but a large molar volume, which indicates a strong binding between atoms but a loose structure, respectively.

Table 3. EMTO_CPA calculated bulk modulus $\left(\mathrm{B}_{0}\right)$ and corresponding molar volume $(\mathrm{Vm})$ of the $\mathrm{A}_{66.7} \mathrm{~B}_{33.3}$ sigma phase in both ordered and disordered states at $0 \mathrm{~K}$.

\begin{tabular}{ccccc}
\hline \multirow{2}{*}{$\mathrm{A}_{x} \mathrm{~B}_{\mathrm{y}}$} & \multicolumn{2}{c}{$\mathrm{B}_{0}, \mathrm{Gpa}$} & \multicolumn{2}{c}{$\mathrm{Vm}, 10^{-6} \mathrm{~m}^{3} / \mathrm{mol}$} \\
\cline { 2 - 5 } & ordered & disordered & ordered & disordered \\
\hline $\mathrm{Cr}_{66.7} \mathrm{Co}_{33.3}$ & 251.345 & 242.347 & 6.799 & 6.869 \\
$\mathrm{Cr}_{66.7} \mathrm{Fe}_{33.3}$ & 257.813 & 249.913 & 6.801 & 6.855 \\
$\mathrm{Cr}_{66.7} \mathrm{Mn}_{33.3}$ & 255.737 & 250.914 & 6.921 & 6.950 \\
$\mathrm{~V}_{66.7} \mathrm{Co}_{33.3}$ & 206.275 & 195.199 & 7.467 & 7.566 \\
$\mathrm{~V}_{66.7} \mathrm{Fe}_{33.3}$ & 212.796 & 203.138 & 7.491 & 7.562 \\
$\mathrm{~V}_{66.7} \mathrm{Mn}_{33.3}$ & 209.324 & 203.167 & 7.643 & 7.675 \\
$\mathrm{~V}_{66.7} \mathrm{Ni}_{33.3}$ & 195.071 & 183.190 & 7.588 & 7.700 \\
$\mathrm{Mo}_{66.7} \mathrm{Oss}_{33.3}$ & 283.269 & 274.343 & 9.478 & 9.491 \\
$\mathrm{Mo}_{66.7} \mathrm{Re}_{33.3}$ & 273.023 & 268.617 & 9.644 & 9.614 \\
$\mathrm{Nb}_{66.7} \mathrm{Ir}_{33.3}$ & 222.794 & 207.189 & 10.345 & 10.417 \\
$\mathrm{Mo}_{66.7} \mathrm{Co}_{33.3}$ & 246.252 & 225.691 & 8.482 & 8.771 \\
$\mathrm{Mo}_{66.7} \mathrm{Mn}_{33.3}$ & 250.635 & 232.845 & 8.633 & 8.808 \\
$\mathrm{Re}_{66.7} \mathrm{Mn}_{33.3}$ & 330.679 & 311.432 & 8.310 & 8.539 \\
$\mathrm{Re}_{66.7} \mathrm{Cr}_{33.3}$ & 319.851 & 306.083 & 8.493 & 8.706 \\
$\mathrm{Re}_{66.7} \mathrm{~V}_{33.3}$ & 296.475 & 288.341 & 8.794 & 8.994 \\
$\mathrm{Nb}_{66.7} \mathrm{Al}_{33.3}$ & 151.447 & 136.561 & 10.493 & 10.870 \\
$\mathrm{Ta}_{66.7} \mathrm{Al}_{33.3}$ & 169.286 & 152.224 & 10.505 & 10.944 \\
\hline & & & & \\
\hline & & &
\end{tabular}

\section{Conclusion}

The influence of atomic order on both the enthalpy of formation and bulk modulus of the sigma phase has been studied by using the EMTO_CPA calculations. At $0 \mathrm{~K}$, the sigma phase in the ordered state bears a low enthalpy of formation, which indicates that the ordered structure is more stable than the less ordered ones. Besides, the sigma phase in the ordered state bears a large bulk modulus, which indicates a strong binding between atoms. On the other hand, we found that the formation enthalpy of the sigma phase depends on the size difference and the electron configuration between the two constitutive elements.

Acknowledgements

This work was supported by the Chinese National Key Project of Science and Technology (Grant number: 2012ZX06004- 
012). W. Liu gratefully acknowledges the financial support from the China Scholarship Council. X.-G. Lu acknowledges the High Performance Computing Center of Shanghai University for providing the calculation facility.

\section{References}

[1] G.-X. Hu, X. Cai, Y.-H. Rong, Fundamentals of materials science, third ed., Shanghai Jiao Tong University Press, Shanghai, 2010.

[2] B. Seiser, R. Drautz, D.G. Pettifor, TCP phase predictions in Ni-based superalloys: Structure maps revisited, Acta Mater. 59 (2011) 749-763. doi:10.1016/j.actamat.2010.10.013.

[3] A.N. Ladines, T. Hammerschmidt, R. Drautz, Structural stability of Fe-based topologically close-packed phases, Intermetallics. 59 (2015) 59-67. doi:10.1016/j.intermet.2014.12.009.

[4] R. Rettig, R.F. Singer, Numerical modelling of precipitation of topologically close-packed phases in nickel-base superalloys, Acta Mater. 59 (2011) 317-327. doi:10.1016/j.actamat.2010.09.035.

[5] M. Palumbo, S.G. Fries, T. Hammerschmidt, T. Abe, J.-C. Crivello, A.A.H. Breidi, J.-M. Joubert, R. Drautz, First-principles-based phase diagrams and thermodynamic properties of TCP phases in Re-X systems (X=Ta, V, W), Comput. Mater. Sci. 81 (2014) 433445. doi:10.1016/j.commatsci.2013.08.051.

[6] J.-Y. Zhao, W. Liu, X.-G. Lu, Assessments of molar volume of the binary C14 Laves phase, Calphad. 50 (2015) 82-91. doi:10.1016/j.calphad.2015.04.011.

[7] E.O. Hall, S.H. Algie, The sigma phase, Metall. Rev. 11 (1966) 61-88.

[8] J.-M. Joubert, Crystal chemistry and Calphad modeling of the $\sigma$ phase, Prog. Mater. Sci. 53 (2008) $528-583$. doi:10.1016/j.pmatsci.2007.04.001.

[9] W. Liu, X.-G. Lu, Y.-L. He, L. Li, Modeling of molar volume of the sigma phase involving transition elements, Comput. Mater. Sci. 95 (2014) 540-550. doi:10.1016/j.commatsci.2014.08.015.

[10] L. Vitos, Computational quantum mechanics for materials engineers: the EMTO method and applications, Springer, London, 2007.

[11] L. Vitos, Total-energy method based on the exact muffin-tin orbitals theory, Phys. Rev. B. 64 (2001). doi:10.1103/PhysRevB.64.014107.

[12] P. Soven, Coherent-potential model of substitutional disordered alloys, Phys. Rev. 156 (1967) 809.

[13] B.L. Gyorffy, Coherent-potential approximation for a nonoverlapping-muffin-tin-potential model of random substitutional alloys, Phys. Rev. B. 5 (1972) 2382.

[14] L. Vitos, I.A. Abrikosov, B. Johansson, Anisotropic Lattice Distortions in Random Alloys from First-Principles Theory, Phys. Rev. Lett. 87 (2001). doi:10.1103/PhysRevLett.87.156401.

[15] J.P. Perdew, K. Burke, M. Ernzerhof, Generalized Gradient Approximation Made Simple, Phys. Rev. Lett. 77 (1996) $3865-3868$. doi:10.1103/PhysRevLett.77.3865.

[16] J. Cieslak, S.M. Dubiel, M. Reissner, Site occupancy and lattice parameters in sigma-phase Co-Cr alloys, Acta Crystallogr. B. 68 (2012) 123-127. doi:10.1107/S0108768112006234.

[17] J. Cieślak, M. Reissner, S.M. Dubiel, J. Wernisch, W. Steiner, Influence of composition and annealing conditions on the siteoccupation in the $\sigma$-phase of Fe-Cr and Fe-V systems, J. Alloys Compd. 460 (2008) 20-25. doi:10.1016/j.jallcom.2007.05.098.

[18] F.J. Spooner, C.G. Wilson, Ordering in binary $\sigma$ phases, Acta Crystallogr. 17 (1964) 1533-1538. doi:10.1107/S0365110X64003826.

[19] S.A. Farzadfar, M. Levesque, M. Phejar, J.-M. Joubert, Thermodynamic assessment of the Molybdenum-Rhenium system, Calphad. 33 (2009) 502-510. doi:10.1016/j.calphad.2009.02.001.

[20] A. Boulineau, J.-M. Joubert, R. Černý, Structural characterization of the Ta-rich part of the Ta-Al system, J. Solid State Chem. 179 (2006) 3385-3393. doi:10.1016/j.jssc.2006.07.001.

[21] W. Liu, X.-G. Lu, P. Boulet, M.-C. Record, Influencing factors of atomic order in the binary sigma phase, Submitted. (n.d.).

[22] J. Cieślak, M. Reissner, W. Steiner, S.M. Dubiel, On the magnetism of the $\sigma$-phase Fe-Cr alloys, Phys. Status Solidi A. 205 (2008) 1794-1799. doi:10.1002/pssa.200723618.

[23] J. Cieślak, B.F.O. Costa, S.M. Dubiel, M. Reissner, W. Steiner, Magnetic ordering above room temperature in the sigma-phase of 
Fe66V34, J. Magn. Magn. Mater. 321 (2009) 2160-2165. doi:10.1016/j.jmmm.2009.01.005.

[24] F.D. Murnaghan, The Compressibility of Media under Extreme Pressures, Proc. Natl. Acad. Sci. 30 (1944) $244-247$.

[25] F. Birch, Finite elastic strain of cubic crystals, Phys. Rev. 71 (1947) 809. 